\title{
Downregulation of miR-143 modulates KRAS expression in colorectal carcinoma cells
}

\author{
HONG LIU ${ }^{1}$, JIANSHENG LIU ${ }^{1}$, JUNKAI HUO ${ }^{2}$, KAIHUA LI $^{2}$, KAI LI $^{2}$, HAOSU GUO $^{2}$ and YIRONG YANG ${ }^{2}$ \\ ${ }^{1}$ Department of General Surgery, First Hospital of Shanxi Medical University; \\ ${ }^{2}$ The First Clinical Medical School, Shanxi Medical University, Taiyuan, Shanxi 030001, P.R. China
}

Received September 30, 2018; Accepted August 14, 2019

DOI: $10.3892 /$ or.2019.7359

\begin{abstract}
MicroRNAs (miRs) are a class of non-coding small RNAs that have been demonstrated to be involved in the pathogenesis of human cancer. There is even evidence that microRNAs can act as oncogenes or tumor suppressors. Although microRNA expression profiles have been characterized in colorectal carcinoma, their precise physiological functions are largely unknown. It has become clear that the activated KRAS Proto-Oncogene, GTPase (KRAS) oncogene plays an important role in colorectal carcinogenesis. In the present study, it was found that the level of mature miR-143 was lower in colorectal carcinoma tissues compared with that observed in normal adjacent tissues. A lack of miR-143 was detected in human colorectal carcinoma cell lines, SW480, LoVo and HT-29, compared to the high expression observed in normal colon epithelial cell line NCM460. pcDNA3.1-pri-miR-143 and its mutant were successfully constructed and transfected into colorectal carcinoma cells. Increased accumulation of mature miR-143 was observed in the pcDNA3.1-pri-miR-143-transfected cells. In SW480 cells, transfection of pcDNA3.1-pri-miR-143 resulted in a 35 and $47 \%$ reduction in cell growth after incubation for 4 and 5 days, respectively, compared with transfection of the pcDNA3.1-pri-miR-143 mutant; while in LoVo cells, transfection of pcDNA3.1-pri-miR-143 resulted in a 33 and $46 \%$ reduction in cell growth respectively. In contrast, transfection of pcDNA3.1-pri-miR-143 had no significantly effects on HT-29 cell growth. We also found that transfection of pcDNA3.1-pri-miR-143 had no effect on levels of KRAS mRNA, but resulted in a $58 \%$ decrease in the KRAS protein level in the transfected SW480 cells, while an approximate 54 and $43 \%$ KRAS protein reduction in LoVo and HT-29 cells, respectively, compared with the pcDNA3.1-pri-miR-143 mutant. Two fragments containing the
\end{abstract}

Correspondence to: Dr Hong Liu, Department of General Surgery, First Hospital of Shanxi Medical University, 85 Jiefangnan Road, Taiyuan, Shanxi 030001, P.R. China

E-mail: 1honglli@sina.cn

Key words: miRNA, miR-143, colorectal carcinoma cells, KRAS putative complementary site were cloned into the pGL3 vector, constructing the luciferase reporter pGL3-KRAS-CS1 and pGL3-KRAS-CS2. Cotransfection of pcDNA3.1-pri-miR-143 with pGL3-KRAS-CS1 and pGL3-KRAS-CS2 respectively resulted in 4.6- and 3.3-fold inhibition of luciferase activity in the SW480 cells, while a 4.0- and 3.2-fold inhibition of luciferase activity in the LoVo cells, 3.7- and 3.1-fold inhibition in the HT-29 cells. Differences in pGL3-KRAS-CS1 and pGL3-KRAS-CS2 activity were not significant. Our results revealed that increased accumulation of miR-143 is likely to modulate levels of KRAS protein expression at the post-transcriptional level by interacting specifically with the complementary site, and consequently inhibiting proliferation of the transfected cells.

\section{Introduction}

Colorectal cancer (CRC) is one of the most highly malignant cancers worldwide and is associated with high morbidity and mortality rate $(1,2)$. CRC evolves through multiple distinct pathways, including the classical adenoma-carcinoma sequence and the serrated pathways (3). These pathways are defined based on molecular features and the pathology of the precursor lesions. The classical adenoma-carcinoma sequence originates in conventional adenomas (tubular adenomas and tubulovillous adenomas), whereas the serrated pathway develops in serrated polyps (SPs). Molecularly, the classical adenoma-carcinoma sequence pathway is characterized by chromosomal instability (CIN) and APC regulator Of WNT signaling pathway (APC) or KRAS proto-oncogene, GTPase (KRAS) mutations (4), whereas in the serrated pathway the genetic alterations include specific B-Raf proto-oncogene, serine/threonine kinase (BRAF) and KRAS mutations, microsatellite instability (MSI), and a CpG island methylator phenotype (CIMP) $(5,6)$. KRAS mutations are found in $35-42 \%$ of CRCs and advanced adenomas (7). Proteins expressed by KRAS genes are involved in the RAF/MEK/ERK mitogen-activated protein kinase (MAPK) signaling pathway, which is a downstream pathway of epidermal growth factor receptor (EGFR). Mutations in KRAS and BRAF genes lead to the persistent activation of this pathway and accelerate the proliferation of tumor cells (8). It has been widely accepted that KRAS mutations predict the poor efficacy of anti-EGFR therapy in patients with metastatic CRC (9). Previous studies 
indicate that KRAS mutations are linked with statistically significant reductions in overall survival and disease-free survival of CRC patients $(10,11)$.

MicroRNAs (miRNAs or miRs) are endogenous RNA molecules 19-25 nucleotides in length, that regulate the expression of genes involved in cell differentiation, proliferation and apoptosis (12-15). A number of studies have demonstrated that altered expression of specific miRNAs contributes to the initiation and progression of CRC (16-18). There is also evidence that miRNAs can act as tumor suppressors or oncogenes depending on the cellular environment in which they are expressed $(19,20)$. In an effort to uncover the relationship between miRNA expression and carcinogenesis, it was found that expression levels of miRNAs are correlated with proto-oncogenes characteristically expressed in CRC. It was demonstrated that miR-192 and miR-215 are both effectors and regulators of p53 function to suppress colon carcinogenesis (21). Another p53-related miRNA, miR-34a, was shown to inhibit cell invasion in colon cancer cell lines by targeting FRA1 (22). miR-320b can target c-Myc in human CRC cells to suppress proliferation (23). Another study showed that c-Myc was able to regulate the miR-17 cluster, and modulate E2F1 expression (24). Johnson et al (25) reported that the RAS oncogene is regulated by the let-7 miRNA family, and decreased expression of let-7 miRNA in various types of human lung tumors caused increased expression of RAS protein. miR-155 was found to be positively regulated by $\mathrm{CBX} 7$ in colon carcinomas, and targets the KRAS oncogene (26).

miR-143 was found to be tissue-specifically expressed in mice (27). It was observed that expression of mature miR-143 is frequently downregulated in approximately $80 \%$ of human colorectal tumor samples from cancer and adenoma patients compared with its level in normal tissues (28). miR-143 can regulate the cell growth and proliferation of CRC in vitro by targeting different oncogenic protein-coding genes, and directly repressing translation of extracellular signal-regulated kinase-5 (ERK5) (29) and DNA (cytosine-5)-methyltransferase $3 \mathrm{~A}(30)$, but the specific mechanism requires further investigation.

In the present study, the miR-143 expression plasmid was constructed and transfected into human colorectal carcinoma cell lines (SW480, LoVo and HT-29). It was found that increased accumulation of miR-143 suppressed expression of KRAS protein in the transfected cells. Furthermore, luciferase reporter assay demonstrated that miR-143 was likely to regulate translation of target mRNAs by hybridization to complementary sites at the $3^{\prime}$ untranslated region (UTR).

\section{Materials and methods}

Tissue samples and cell lines. Colorectal tumors and the corresponding normal mucosa were obtained from fresh surgical excision at the First Hospital of Shanxi Medical University, Taiyuan, China from May 20 to July 26, 2018. This study was approved by the Ethics Commission of the First Hospital of Shanxi Medical University [approval no. 2018(k012), Taiyuan, China)]. Informed consent was obtained from all patients before surgery. A total of 8 patients, including 5 males and 3 females were recruited. The age range was 41-63 years, with an average age of $51.8 \pm 6.72$ years. Histopathological examination of each sample was performed to confirm its diagnostic yield. The tissues were placed in liquid nitrogen immediately after resection and stored at $-80^{\circ} \mathrm{C}$. Human colorectal carcinoma cell lines SW480 (KRAS mutant-type), LoVo (KRAS mutant-type), HT-29 (KRAS wild-type) and normal colon epithelial cell line NCM460 were obtained from the American Type Culture Collection (ATCC; Manassas, VA, USA). The cells were cultured in Dulbecco's modified Eagle's medium (DMEM) (Gibco; Thermo Fisher Scientific, Inc.) supplemented with $10 \%$ heat-inactivated fetal bovine serum (Sigma-Aldrich; Merck $\mathrm{KGaA}$ ) at $37^{\circ} \mathrm{C}$ in a humidified atmosphere with $5 \% \mathrm{CO}_{2}$.

$R N A$ isolation and quantitative reverse transcription polymerase chain reaction ( $R T-q P C R)$. Total RNA was extracted from the cultured cells and tissues using TRIzol reagent (Invitrogen; Thermo Fisher Scientific, Inc.). The mature miR-143 was assessed using TaqMan miRNA Real-Time PCR assays and the Rotor-Gene 3000 thermal cycler (Qiagen, Shanghai, China). Briefly, $1 \mu \mathrm{g}$ total RNA was reverse-transcribed to cDNA using AWV reverse transcriptase (Takara, Dalian, China), and the expression of miR-143 was measured by RT-qPCR. The sequences of the primers (Sangon Biotech Co., Ltd., Shanghai, China) were: miR-143 forward, 5'-GGGTGAGATGAAGCACTGTAGCTC-3' and reverse, 5'-GCTGTCAACATACGCTACGTAACG-3'; human U6 forward, 5'-CGCTTCACGAATTTGCGTGTCA-3' and reverse, 5'-GCTTCGGCAGCACATATACTAAAAT-3'. The reaction conditions were as follows: $95^{\circ} \mathrm{C}$ for $1 \mathrm{~min}$, followed by 40 cycles at $95^{\circ} \mathrm{C}$ for $30 \mathrm{sec}, 60^{\circ} \mathrm{C}$ for $30 \mathrm{sec}, 95^{\circ} \mathrm{C}$ for $45 \mathrm{~min}, 55^{\circ} \mathrm{C}$ for $1 \mathrm{~min}$ and $55^{\circ} \mathrm{C}$ for $10 \mathrm{sec}$. U6 small nuclear (sn)RNA was used as an internal control, and the relative expression levels of miR-143 were calculated by the $2^{-\Delta \Delta \mathrm{Cq}}$ method (31).

Cloning of pri-miR-143 and plasmid construction. Genomic DNA was extracted using lysis buffer containing $10 \mathrm{mM}$ Tris- $\mathrm{HCl}, \mathrm{pH} 8.0 ; 100 \mathrm{mM} \mathrm{NaCl} ; 0.5 \%$ SDS; $25 \mathrm{mM}$ EDTA, treated with proteinase $\mathrm{K}(0.2 \mathrm{mg} / \mathrm{ml})$, purified with phenol/chloroform, precipitated with ethanol and dissolved in DNase-free water. RT-PCR was performed using the following primers (32): 5'-AGGTTTGGTCCTGGGTGCTCA AATGGCAGG-3' (forward) and 5'-TGCCCAGACTCGTGA AGCAGATCGTGGCAC-3' (reverse). pGEM-pri-miR-143 was constructed by T/A cloning of a 430-bp pri-miR-143 PCR product into the pGEM-T-Easy vector (Promega). Mutant pri-miR-143 was derived from pGEM-pri-miR-143 using the QuickChange method (Stratagene). The reaction was performed in the presence of $p f u$ hotstart DNA polymerase (Stratagene) for 18 cycles at $95^{\circ} \mathrm{C}$ for $1 \mathrm{~min}, 60^{\circ} \mathrm{C}$ for $1 \mathrm{~min}$ and $68^{\circ} \mathrm{C}$ for 8 min using PTC-100 thermocycler (MJ-Research). The mutagenic primers were as follows: 5'-TGGTCAGTTGGG AGTCAGCACTGTAGCTCAGG-3' (forward) and 5'-CCT GAGCTACAGTGCTGACTCCCAACTGACCA-3' (reverse). pcDNA3.1-pri-miR-143 and pcDNA3.1-pri-miR-143m were constructed by subcloning pri-miR-143 and mutated pri-miR-143 into pcDNA3.1 eukaryotic expression vector (Invitrogen), respectively. The DNA insert from the recombinant plasmid was sequenced by chain-termination dideoxy sequencing to confirm the mutation. 
Transfection in vitro and cell proliferation assay. Transient transfections were performed using Lipofectamine 2000 (Invitrogen; Thermo Fisher Scientific, Inc.). Human colorectal carcinoma cell lines SW480, LoVo or HT-29 cells were plated in duplicate 1 day before transfection, so that the cells were 90-95\% confluent at the time of transfection. We used $1.6 \mu \mathrm{g}$ plasmid DNA (expression plasmid pcDNA3.1-pri-miR-143, pcDNA3.1-pri-miR-143 mutant or empty vector pcDNA3.1) for each well of a 12-well plate. After $5 \mathrm{~h}$ of incubation, DNA-Lipofectamine 2000 complexes were removed and replaced by normal medium. Cells were used for experiment $24 \mathrm{~h}$ after transfection.

Cell proliferation was evaluated by colorimetric 3-(4,5-dimethylthiazol-2-yl)-2,5-diphenyltetrazolium bromide (MTT) assay. Transfected SW480, LoVo and HT-29 cells were respectively seeded in 96-well plates in triplicate and incubated for 1-5 days. A 1/10 volume of MTT solution (4 mg/ml in PBS) was added and incubated for $4 \mathrm{~h}$ at $37^{\circ} \mathrm{C}$. The supernatant was aspirated, and an equal volume of dimethyl sulfoxide was added to the cells. MTT formazan was dissolved by pipetting. The absorbance was measured on an ELISA plate reader (Bio-Rad) at a wavelength of $570 \mathrm{~nm}$. All the experiments were repeated at least three times.

RT-qPCR analysis. To quantify the KRAS mRNA, total RNA was isolated 48 or $72 \mathrm{~h}$ after transfection using TRIzol reagent (Invitrogen; Thermo Fisher Scientific, Inc.). Total RNA $(1 \mu \mathrm{g})$ was reverse-transcribed to cDNA using oligo(dT) (Takara) and AMV reverse transcriptase (Takara). The reaction conditions were: $16^{\circ} \mathrm{C}$ for $30 \mathrm{~min}, 42^{\circ} \mathrm{C}$ for $30 \mathrm{~min}$ and $85^{\circ} \mathrm{C}$ for $5 \mathrm{~min}$. Real-time PCR was then processed using the RT product, SYBR Green dye (Invitrogen; Thermo Fisher Scientific, Inc.) and specific primers for KRAS and $\beta$-actin. The relative amount of KRAS mRNA was normalized to $\beta$-actin. The sequences of the primers were as follows: KRAS (sense), 5'-GACTCTGAAGATGTACCTATGGTCCTA-3' and KRAS (antisense), 5'-CATCATCAACACCCTGTCTTGTC-3'; $\beta$-actin (sense), 5'-TCACCCACACTGTGCCCATCTACGA-3' and $\beta$-actin (antisense), 5'-CAGCGGAACCGCTCATTGCCA ATGG-3'. The reactions were incubated at $95^{\circ} \mathrm{C}$ for $5 \mathrm{~min}$, followed by 40 cycles of $95^{\circ} \mathrm{C}$ for $30 \mathrm{sec}, 60^{\circ} \mathrm{C}$ for $30 \mathrm{sec}$ and $72^{\circ} \mathrm{C}$ for $30 \mathrm{sec}$. The results were confirmed by three independent experiments.

Western blotting. Total protein was extracted from tissue samples and cells with protein extraction reagent (Pierce; Thermo Fisher Scientific, Inc.). The protein concentration was determined using a BCA Protein Assay Reagent kit (Pierce; Thermo Fisher Scientific, Inc.). Total protein $(10 \mu \mathrm{g})$ was separated on $12 \%$ SDS-PAGE and electrotransfered to polyvinylidene fluoride membranes. Primary antibodies binding to KRAS (mouse monoclonal antibody, cat. no. sc-30; 1:5,000 dilution; Santa Cruz Biotechnology), NRAS (mouse monoclonal antibody, cat. no. sc-519; 1:5,000 dilution; Santa Cruz Biotechnology), $\beta$-actin (mouse monoclonal antibody, cat. no. A5316; 1:1,000 dilution; Sigma-Aldrich; Merck KGaA) were detected with horseradish-peroxidase-conjugated anti-mouse secondary antibodies (cat. no. AP308P; 1:2,000 dilution; Sigma-Aldrich; Merck KGaA) and the ECL Plus Western Blotting Detection kit (Amersham).
Scanned images were quantified using Quantity One software (version 4.6.9; Bio-Rad Laboratories).

Luciferase assay. Two KRAS cDNA fragments, containing putative miR-143 complementary site (CS), were amplified by RT-PCR with the following primer sets: KRAS-CS1, 5'-TTA CAATCTCTAGGTTTGGCTAGTTCTC-3' (forward) and 5'-GTCTAGAAGGTAGGGAGGCAAGATGAC-3' (reverse); KRAS-CS2, 5'-GCCTCT TGA ATTTTTGATGTAGAT G-3' (forward) and 5'-GTCTAGACAAATGGAAATCTT CAGATA-3' (reverse). pGL3-KRAS-CS was constructed by insertion of $X b a \mathrm{I}$-digested KRAS-CS in the $X b a \mathrm{I}$ site of the pGL3 control vector (Promega). Various pGL3 reporters were constructed, and then cotransfected into the CRC cells with pcDNA3 plasmids using Lipofectamine 2000 transfection reagent. After 48 and $72 \mathrm{~h}$ of transfection, the luciferase and $\beta$-galactosidase activities were determined.

Statistical analysis. All of the western blotting and proliferation assay images are representative of at least three independent experiments. RT-qPCR and luciferase reporter assays were performed in triplicate. SPSS 20.0 software (IBM Corp., Armonk, NY, USA) was used for statistical analysis, and the data are presented as the mean \pm standard error of the mean. Statistical analysis was performed using Student's t-test or one-way ANOVA followed by the Dunnett's t test. $\mathrm{P}<0.05$ was considered indicative of a statistically significant result.

\section{Results}

Reduced expression of miR-143 in colorectal neoplasia. Total RNA was isolated from colorectal carcinoma tissues, normal adjacent mucosa and cell lines. snRNA was examined to confirm its high quality by staining with ethidium bromide (Fig. 1A). RT-qPCR assay was performed to validate the expression levels of mature miR-143 in cell lines. The results showed a lack of miR-143 in human colorectal carcinoma cells SW480, LoVo and HT-29, compared to high expression in normal colon epithelial cell line NCM460 (Fig. 1B). The same method revealed an $\sim 53.2 \%$ in reduction of the miR-143 level in tumors compared with that noted in the normal adjacent tissues (Fig. 1C). This observation was consistent with the findings of a previous study reporting reduction of miR-143 in colorectal neoplasia (28).

Accumulation of miR-143 in the transfected cells. Expression plasmid pcDNA3.1-pri-miR-143 was constructed. The 430-bp DNA insert was sequenced (Fig. 2A), and compared with the GenBank database (NT_029289). A pcDNA3.1-pri-miR-143 mutant was derived by knockdown of 8 nucleotides (TGAGATGA) at the 5'end of miR-143, which was a putative binding site (Fig. 2B). The mutated plasmid pcDNA3.1-pri-miR-143m was utilized as a control in research of miR-143 function. To investigate whether changes in miR-143 expression levels were associated with cell proliferation and tumorigenesis, we transfected pcDNA3.1, pcDNA3.1-pri-miR-143 or pcDNA3.1-pri-miR-143m into SW480, LoVo and HT-29 cells respectively. The mRNA levels of pri-miR-143 or pri-miR-143 mutant in the transfected cells were detected by RT-PCR, Subsequently the 

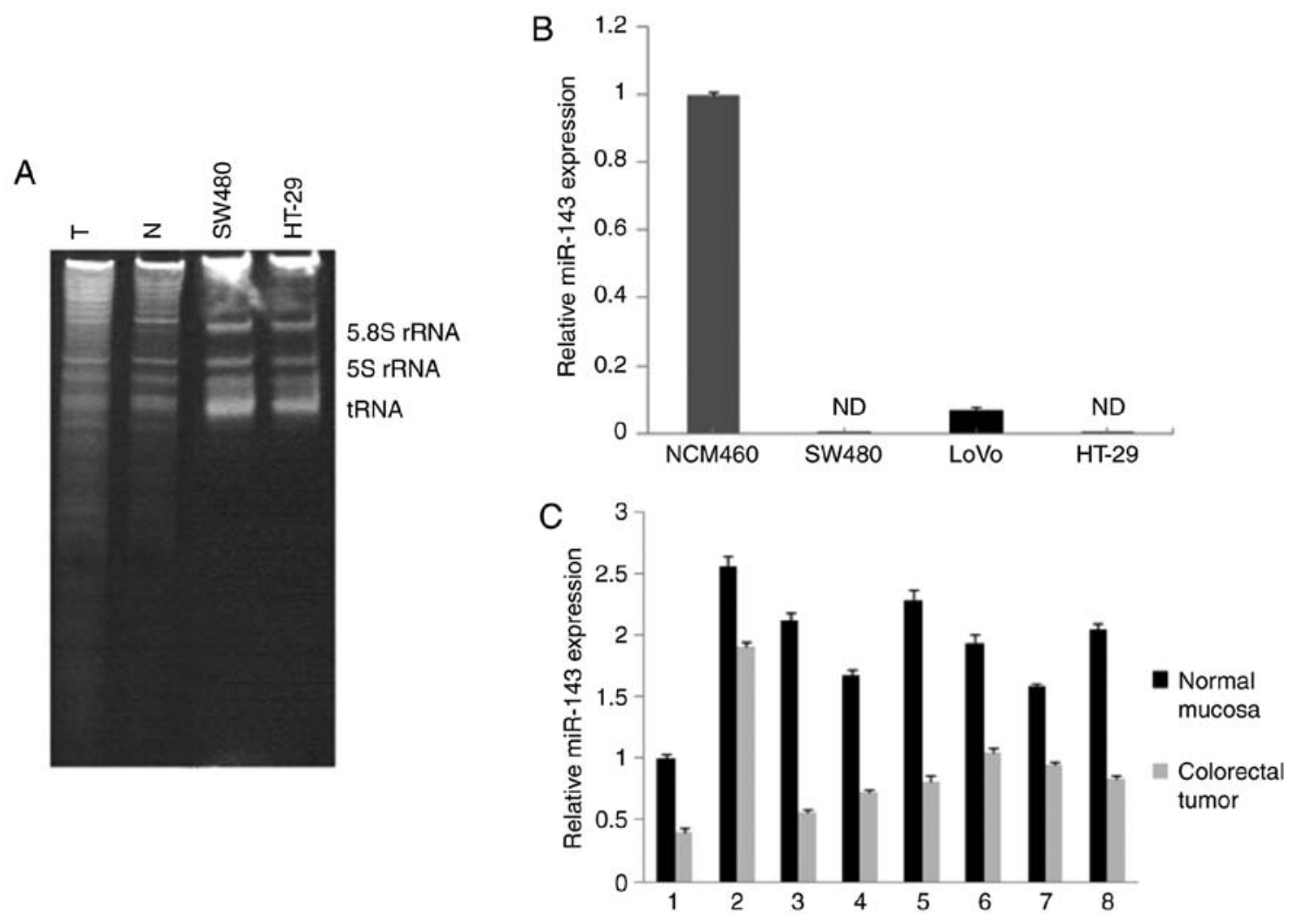

Figure 1. Expression levels of mature miR-143 in colorectal tissues and cell lines. (A) Total RNA (10 $\mu \mathrm{g})$ from colorectal tumors (T), normal adjacent mucosa (N) or cell lines was separated on denaturing 15\% polyacrylamide gels, and stained with ethidium bromide. Clearly visible tRNA, 5S rRNA and 5.8S rRNA demonstrated high quality of small RNAs. (B) RT-qPCR analysis of mature miR-143 levels in CRC. The bars indicate relative miR-143 expression in SW480, LoVo and HT-29 cell lines when normal colon epithelial cell line NCM460 was set as 1. U6 snRNA served as an endogenous control. ND indicates not detected. (C) RT-qPCR analysis of mature miR-143 levels in 8 colorectal tissue pairs. Bars 1-8 indicate relative miR-143 expression in colorectal tumor and corresponding normal adjacent mucosa when the normal mucosa in the first colorectal tissue pair was set to 1. Error bars for all panels represent standard deviations derived from at least three independent measurements. CRC, colorectal cancer.

product was sequenced to confirm the efficiency of gene transfection. The results showed the significantly increased expression of pri-miR-143 in the transfected cells with pcDNA3.1-pri-miR-143, related to the empty vector, and transfection of pcDNA3.1-pri-miR-143m was confirmed by gene sequencing (Fig. 2C). RT-qPCR was used to detect the level of mature miR-143. Increased accumulation of mature miR-143 was found in the pcDNA3.1-pri-miR-143-transfected cells, while transfection of pcDNA3.1-pri-miR-143m had no effects on the level of miR-143 (Fig. 2D). It should be emphasized that expression of the miR-143 mutant was not measured by RT-qPCR, due to its poor specificity (14 base-fragment, 3'-ACTCGATGTCACGA-5').

Effects of miR-143 on cell proliferation. miRNAs are involved in cell proliferation and differentiation. Therefore, we examined the effects of miR-143 transfection on the proliferation of CRC cells. The cells transfected with pcDNA3.1-pri-miR-143, pcDNA3.1-pri-miR-143 mutant or pcDNA3.1 were incubated for 1-5 days before measuring absorbance by colorimetric MTT assay. In SW480 cells, transfection of pcDNA3.1-pri-miR-143 resulted in 35 and $47 \%$ reduction in cell growth after incubation for 4 and 5 days, respectively, compared with transfection of pcDNA3.1-pri-miR-143m (Fig. 3A), while in LoVocells, transfection of pcDNA3.1-pri-miR-143 resulted in 33 and $46 \%$ reduction in cell growth after incubation for 4 and 5 days, respectively
(Fig. 3B). In contrast, transfection of pcDNA3.1-pri-miR-143 had no significantly effects on HT-29 cell growth (Fig. 3C). This demonstrated that increased accumulation of miR-143 is capable of inhibiting the proliferation of KRAS-mutant cell lines, but had no effects on KRAS wild-type cell lines.

miR-143 partially suppresses KRAS protein expression. There have been several efforts to use bioinformatics techniques to identify miRNA target mRNAs. KRAS transcript was one of the predicted miR-143 targets $(33,34)$. When expression plasmids were transfected into SW480, LoVo and HT-29 cells, respectively, increased accumulation of miR-143 was achieved only in cells transfected with pcDNA3.1-pri-miR-143 (Fig. 2D). We observed an $\sim 58 \%$ decrease in KRAS protein levels in the pcDNA3.1-pri-miR-143-transfected SW480 cells, but not in the pcDNA3.1-pri-miR-143m-transfected cells (Fig. 4B and C). Similarly, transfection with pcDNA3.1-pri-miR-143 resulted in approximately 54 and $43 \%$ KRAS protein reduction in the LoVo and HT-29 cells, respectively, compared with pcDNA3.1-pri-miR-143m (Fig. 4D-G). As a control for target specificity, expression of NRAS protein was not affected (Fig. 4B-G). We also found that levels of KRAS mRNA were not affected by transfection of miR-143 (Fig. 4A). These results suggest that increased accumulation of miR-143 specifically suppresses KRAS protein expression at the post-transcriptional level. 

A 270
280
290
B 270
280

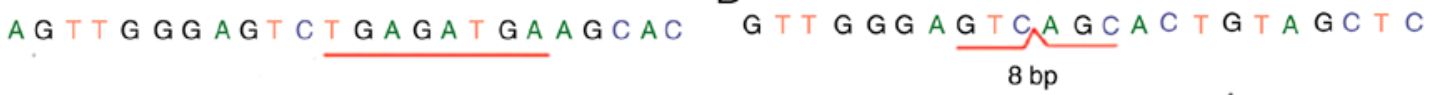
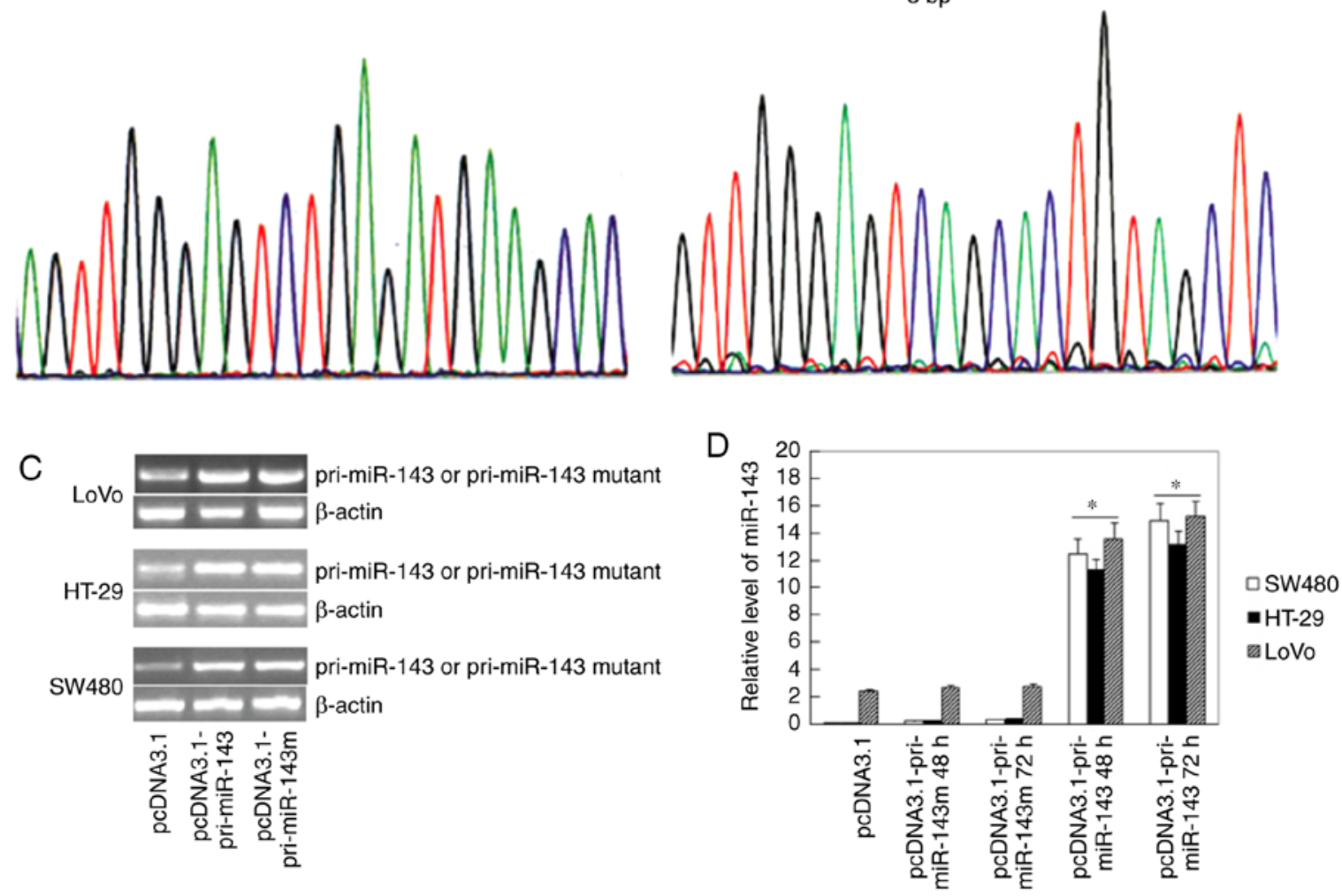

Figure 2. miR-143 plasmid constructs and accumulation of miR-143 in transfected cells. (A) Sequence analysis of pcDNA3.1-pri-miR-143. Red underline shows 8 nucleotides at the 5 'end of miR-143, which is a putative binding site. (B) Sequence analysis of pcDNA3.1-pri-miR-143 mutant. Knockdown of putative binding site was achieved. (C) mRNA levels of pri-miR143 or pri-miR143 mutant in transfected CRC cells. $\beta$-actin served as an endogenous control. Subsequently the RT-PCR product was sequenced. (D) Expression of mature miR-143 in transfected SW480, LoVo or HT-29 cells; empty vector pcDNA3.1 and pcDNA3.1-pri-miR-143 mutant were used as controls. "P<0.05. CRC, colorectal cancer.
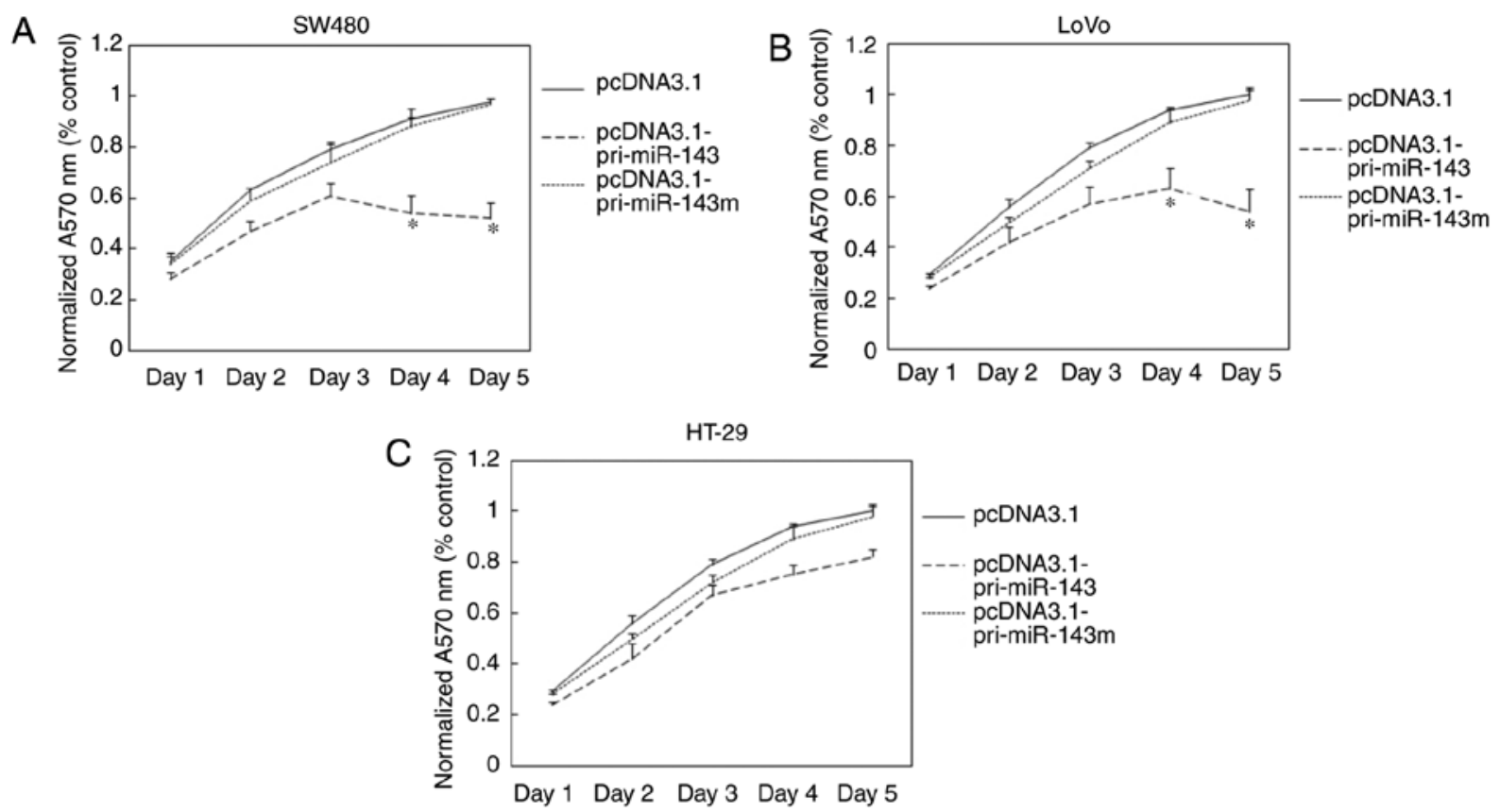

Figure 3. Inhibition of CRC cell growth by miR-143 transfection. Transfected SW480, LoVo and HT-29 cells were incubated for 1-5 days, and absorbance was determined at $570 \mathrm{~nm}$ using MTT assay. The plots show mean \pm SD of triplicate measurements. (A) Proliferation was inhibited in SW480 cells transfected with pcDNA3.1-pri-miR-143 after incubation for 1-5 days, when compared with cells transfected with pcDNA3.1-pri-miR-143m. (B) Proliferation was inhibited in LoVo cells transfected with pcDNA3.1-pri-miR-143 after incubation for 1-5 days, when compared with cells transfected with pcDNA3.1-pri-miR-143m. (C) Proliferation was not significantly affected in HT-29 cells transfected with pcDNA3.1-pri-miR-143 after incubation for 1-5 days, when compared with cells transfected with pcDNA3.1-pri-miR-143m. "P<0.05 (Dennett's t-test). CRC, colorectal cancer. 
A

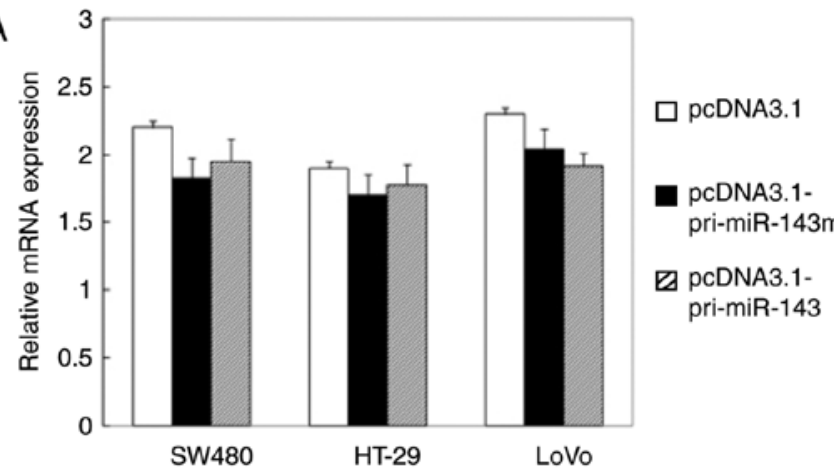

B

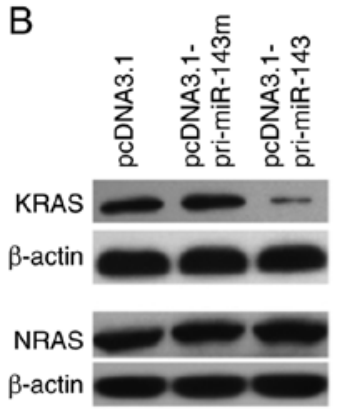

SW480 72 h

D

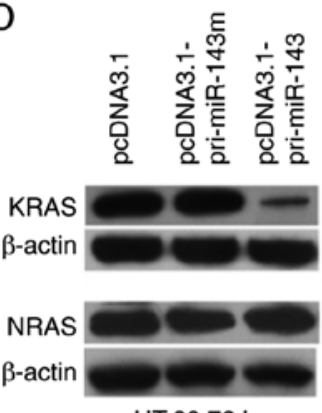

HT-29 72 h

$\mathrm{F}$

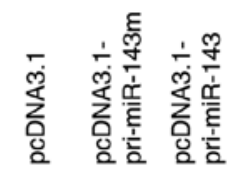

KRAS

$\beta$-actin

NRAS

$\beta$-actin

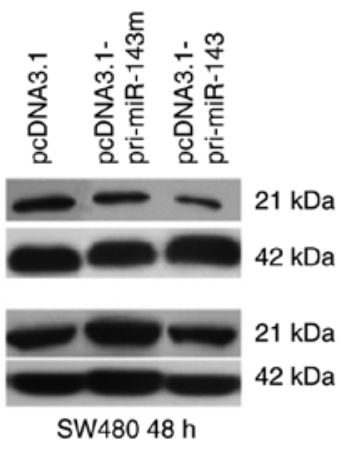

SW480 $48 \mathrm{~h}$



E
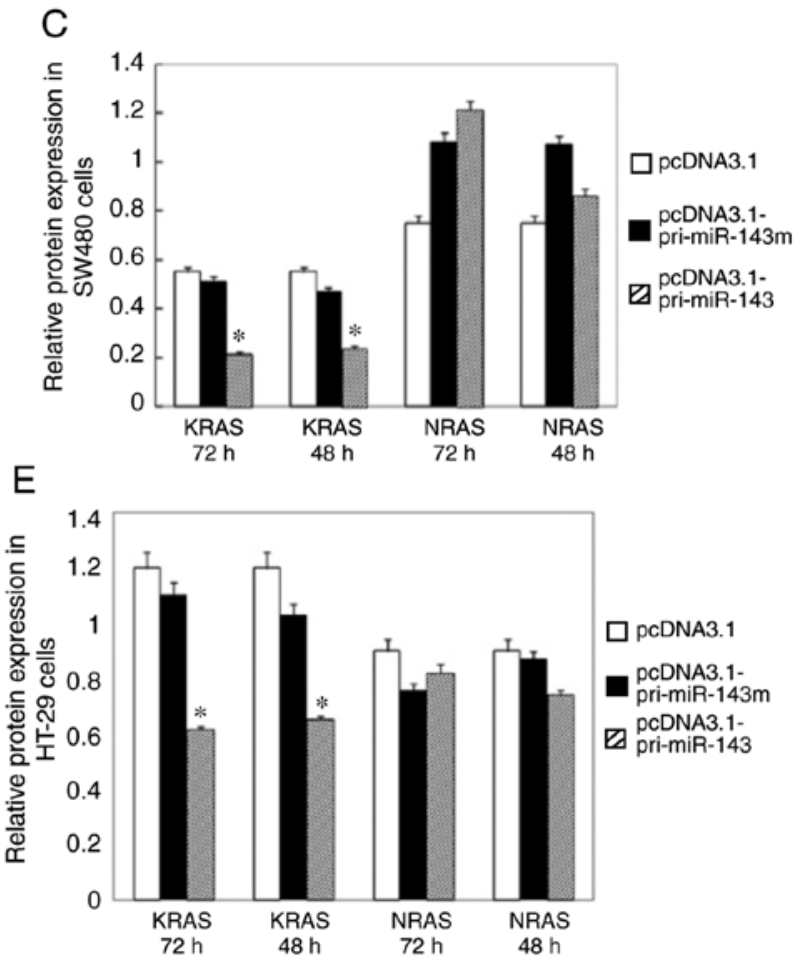

G

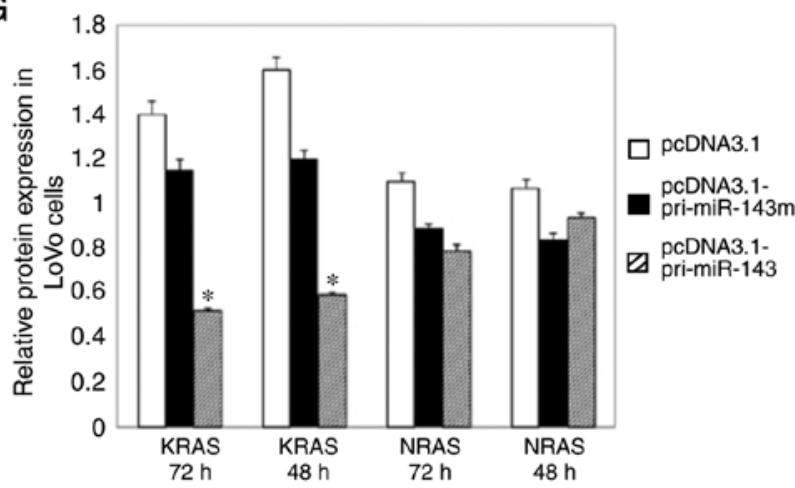

Figure 4. miR-143 partially suppresses KRAS protein expression without altering mRNA abundance. (A) RT-qPCR analysis of KRAS mRNA levels in transfected $\mathrm{CRC}$ cells $72 \mathrm{~h}$ after transfection. $\beta$-actin served as an endogenous control. Normalization to the control, allowing comparison of mRNA levels. (B) Western blotting of KRAS and NRAS proteins in SW480 cells transfected with pcDNA3.1-pri-miR-143, pcDNA3.1-pri-miR-143m and pcDNA3.1 48 and $72 \mathrm{~h}$ after transfection. $\beta$-actin was detected as a loading control. (C) Normalization to the control, allowing comparison of protein expression in transfected SW480 cells. (D) Western blotting of KRAS and NRAS proteins in HT-29 cells transfected with pcDNA3.1-pri-miR-143, pcDNA3.1-pri-miR-143m and pcDNA3.1 48 and $72 \mathrm{~h}$ after transfection. (E) Normalization to the control, allowing comparison of protein expression in transfected HT-29 cells. (F) Western blotting of KRAS and NRAS proteins in LoVo cells transfected with pcDNA3.1-pri-miR-143, pcDNA3.1-pri-miR-143m and pcDNA3.1 48 and $72 \mathrm{~h}$ after transfectinon. (G) Normalization to the control, allowing comparison of protein expression in transfected LoVo cells. Error bars for all panels represent SDs derived from at least three independent measurements. " $\mathrm{P}<0.05$. CRC, colorectal cancer; KRAS, KRAS proto-oncogene, GTPase; NRAS, NRAS proto-oncogene, GTPase.

Putative miR-143 complementary sites in the 3'UTRs of the KRAS gene. It is believed that miRNAs inhibit expression of target mRNAa by interacting with imperfect target sites in the 3'UTR (Fig. 5B). A putative binding site was 
A

$5^{\prime}$

3'

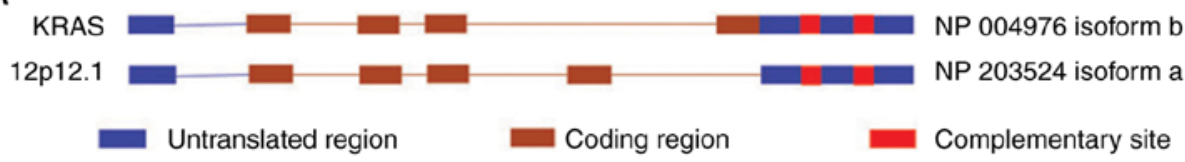

B Homo sapiens miR-143: 3'-ACTCGATGTCACGAAGTAGAGT-5'

KRAS-3'UTR-1:

|l||||

KRAS-3'UTR-2:

4501-GTTTGCACAAGTTCATCTCATTTGTATTCC-4530

2459-TGTTAAAAGAAGTCATCTCAAACTCTTAGT-2488
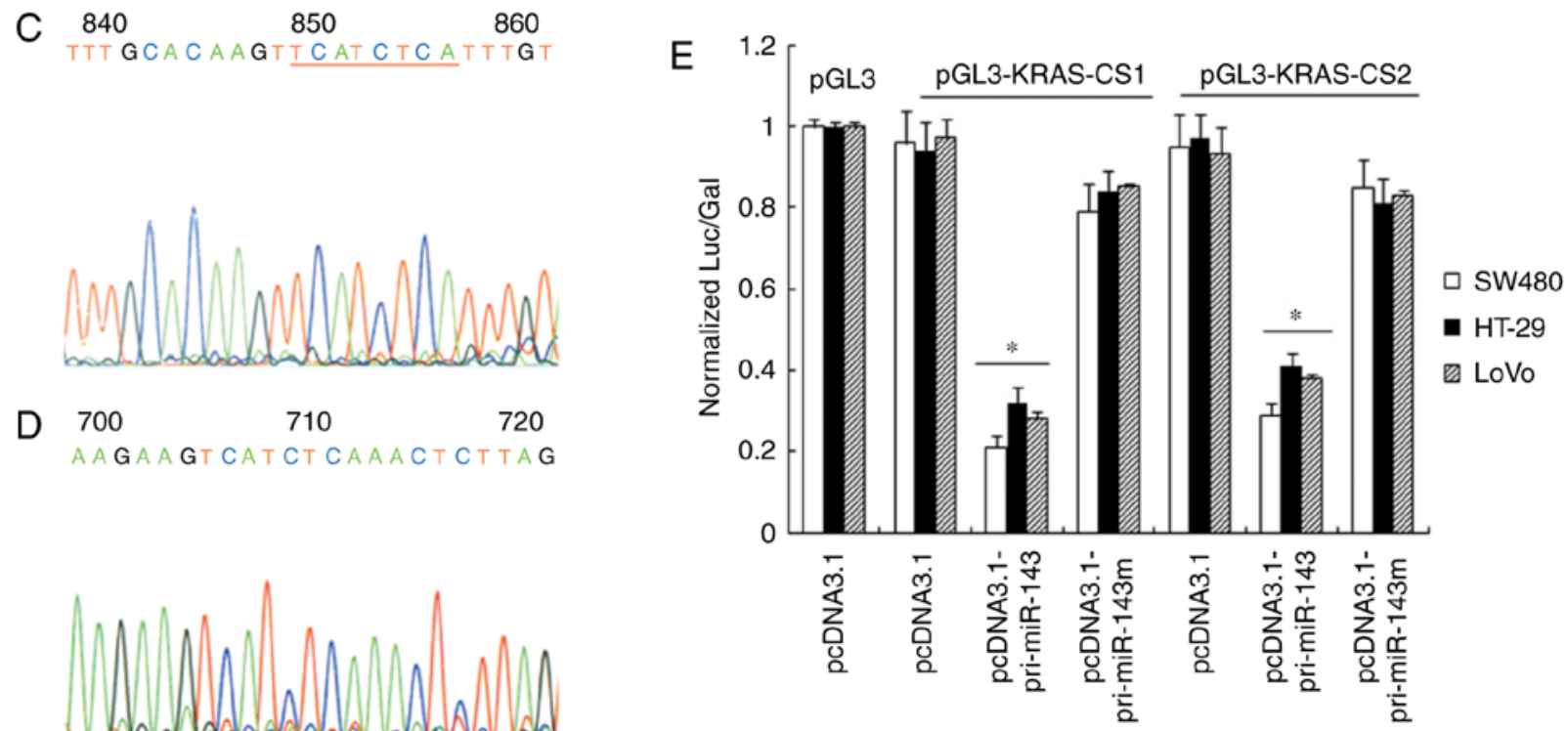

Figure 5. miR-143 interacts specifically with the complementary site of KRAS mRNA 3'UTRs. (A) Schematic representation of the KRAS genomic regions and transcripts. (B) KRAS mRNA 3'UTRs have two putative miR-143 complementary sites. (C) Sequence analysis of luciferase reporter construct 1 (pGL3-KRAS-CS1). (D) Sequence analysis of luciferase reporter construct 2 (pGL3-KRAS-CS2). Underline shows the putative complementary site. (E) SW480, LoVo and HT-29 cells were transiently cotransfected with miR-143 expression plasmid $(0.8 \mu \mathrm{g} / \mathrm{ml})$ together with the luciferase reporter (Luc) and $\beta$-galactosidase $(\mathrm{Gal})$ reference plasmids $(0.4 \mu \mathrm{g} / \mathrm{ml}$ each). Luciferase activity was normalized against $\beta$-galactosidase activity. Data represent the mean \pm SD of six independent experiments. ${ }^{*} \mathrm{P}<0.05$ compared with pGL3-KRAS-CS1 or pGL3-KRAS-CS2 transfection alone (Student's t-test). KRAS, KRAS proto-oncogene, GTPase; UTR, untranslated region.

located at the 5'end of miR-143 (TGAGATGA). We found that the human KRAS 3'UTR contained two miR-143 complementary sites, locating at nucleotides 2471-2478 and 4513-4521 (Fig. 5A). Two fragments containing the complementary sites were fused to the $X b a \mathrm{I}$ site of the pGL3 control vector, constructing the luciferase reporter pGL3-KRAS-CS1 and pGL3-KRAS-CS2, respectively (Fig. 5C and D). After transfection, pri-miRNA-143 and pri-miRNA-143m were detected by RT-PCR and gene sequencing, which confirmed the efficiency and reliability of gene transfection. The expression of miR-143 can be measured by RT-qPCR, while the miR-143 mutant is difficult to detect because of its poor specificity. Cotransfection of pcDNA3.1-pri-miR-143 and pGL3-KRAS-CS1 into SW480 cells resulted in 4.6-fold inhibition of luciferase activity compared with pGL3-KRAS-CS1 transfection alone, while cotransfection of pcDNA3.1-pri-miR-143 and pGL3-KRAS-CS2 resulted in 3.3-fold inhibition compared with pGL3-KRAS-CS2 transfection alone. We also found a 4.0- and 3.2-fold inhibition of luciferase activity in LoVo cells, while a 3.7- and 3.1-fold inhibition in HT-29 cells, when pcDNA3.1-pri-miR-143 was cotransfected with
pGL3-KRAS-CS1 and pGL3-KRAS-CS2, respectively. Differences in pGL3-KRAS-CS1 and pGL3-KRAS-CS2 activity were not significant. As a control, transfection of pcDNA3.1-pri-miR-143m had no effects on luciferase activity (Fig. 5E). This supported specific miR-143 interaction with complementary sites of KRAS 3'UTR.

\section{Discussion}

We investigated the expression levels of miR-143 in colorectal carcinoma tissue specimens. The results showed a reduction in mature miR-143 in tumor tissues compared with that noted in normal adjacent tissues, which are consistent with previous reports (28). miRNA downregulation in tumor tissue may be a consequence of the undifferentiated state. Several studies have revealed the prognostic significance of miRNA profiles in colorectal cancer (CRC) $(35,36)$. Expression levels of miR-143 are frequently altered in tumors (29,37-39). There is also evidence that miR-143 is related to expression of KRAS in CRC. A novel synthetic miR-143 can strongly silence KRAS, and increase efficacy of epidermal growth factor receptor (EGFR) inhibitors. Downregulation of KRAS-interacting 
miRNA-143 predicts poor prognosis but not response to EGFR-targeted agents in CRC (40).

The RAS protein family plays a key role in regulating diverse cellular pathways important for cell growth, differentiation and survival (41). Constitutive RAS signaling as a result of RAS mutations or overexpression can induce oncogenesis. Activated KRAS protein was observed in $40-50 \%$ of human colorectal adenomas and carcinomas (42). To explore the effects of miR-143 on KRAS, we constructed the pri-miR-143 expression plasmid and mutation was introduced into the predicted binding site. Colorectal carcinoma cell lines SW480 and LoVo were used in experiments in vitro. SW480 cells carry two mutated alleles at codon 12 of the KRAS oncogene, and LoVo cells harbor a KRAS mutation at codon 13 (43). As a control cell line, HT-29 cells with KRAS-wild-type and BRAF mutant were selected. We detected the expression levels of miR-143 in cell lines and found a lack of miR-143 in human colorectal carcinoma cells SW480, LoVo and HT-29, compared to high expression in normal colon epithelial cell line NCM460. Then the plasmids were transfected into colorectal carcinoma cells respectively, and achieved accumulation of mature miR-143. The results showed that KRAS protein expression was partially inhibited in miR-143-transfected cells, compared with that in the mutated-miR-143-transfected cells. However, the protein expression levels of NRAS were unaffected by the increased miR-143, indicating that miR-143 regulation is specific to KRAS. Similar effect of miR-143 on KRAS expression in KRAS-wild-type and KRAS-mutant cell lines indicates that miR-143 interacts with the KRAS untranslated region, but not the translated region. By cell proliferation assay we found that the cell growth of SW480 and LoVo cells was obviously inhibited after miR-143 transfection, probably due to the downregulation of mutant KRAS protein. In contrast, transfection of miR-143 had no significant effects on HT-29 cell growth. There is evidence that blocking KRAS activity in tumor cell lines can result in cell death or reversion to a nonmalignant phenotype $(44,45)$. Another possible explanation is that increased accumulation of miR143 suppresses cell proliferation by regulating ERK5 (32) or IGF1R (46).

miRNAs have been shown to regulate gene expression by inducing mRNA cleavage or translational repression. In animals, most miRNAs bind with imperfect complementarity to multiple sites in the 3'UTR of their target mRNAs and cause translational repression (12). The potential targets of miRNA were predicted by bioinformatic approaches with an emphasis on the critical pairing at the 5'end of the miRNA (47). To demonstrate that miR-143 directly regulates KRAS expression, we identified two putative binding sites at the 3'UTR of KRAS mRNA, which are complementary with the base sequence at the 5'end of miR-143. The two complementary sites were cloned into the pGL3 control vector, constructing the luciferase reporter. Then, the reporter plasmids were cotransfected into CRC cells, respectively. We found that miR-143 negatively regulated luciferase activity compared with mutated miR-143, which indicates that miR-143 can interact specifically with complementary sites of KRAS 3'UTR. The results suggested that miRNA-mediated gene regulation at the post-transcriptional level is involved in tumorigenesis and tumor progression in colorectal neoplasia, which provides a clue for research of the function of miRNAs and their targets.

\section{Acknowledgements}

The authors would like to thank Dr Chenghong Peng (Department of General Surgery, Ruijin Hospital Shanghai Jiao Tong University School of Medicine, Shanghai, China) for his great assistance in the writing of the manuscipt.

\section{Funding}

The present study was supported by the Natural Science Foundation of Shanxi Province in China (grant no. 2010011047-4).

\section{Availability of data and materials}

The datasets used and/or analyzed during the current study are available from the corresponding author on reasonable request.

\section{Authors' contributions}

HL, JH, KaiLi, HG and YY performed the experiments. HL and KaihuaLi analyzed the data. JL guided the experiments and designed the study. HL wrote the manuscript. All authors read and approved the manuscript and agree to be accountable for all aspects of the research in ensuring that the accuracy or integrity of any part of the work are appropriately investigated and resolved. All authors read and approved the final manuscript.

\section{Ethics approval and consent to participate}

This study was approved by the Ethics Commission of First Hospital of Shanxi Medical University [approval no. 2018(k012), Taiyuan, China]. Written informed consent was obtained from all participants included in the study.

\section{Patient consent for publication}

Not applicable.

\section{Competing interests}

The authors declare that they have no competing interests.

\section{References}

1. Siegel RL, Miller KD and Jemal A: Cancer statistics, 2017. CA Cancer J Clin 67: 7-30, 2017.

2. Siegel RL, Miller KD, Fedewa SA, Ahnen DJ, Meester RGS, Barzi A and Jemal A: Colorectal cancer statistics, 2017. CA Cancer J Clin 67: 177-193, 2017.

3. Jass JR: Classification of colorectal canfcer based on correlation of clinical, morphological and molecular features. Histopathology 50: 113-130, 2007.

4. Pino MS and Chung DC: The chromosomal instability pathway in colon cancer. Gastroenterology 138: 2059-2072, 2010.

5. Leggett B and Whitehall V: Role of the serrated pathway in colorectal cancer pathogenesis. Gastroenterology 138: 2088-2100, 2010.

6. Rex DK, Ahnen DJ, Baron JA, Batts KP, Burke CA, Burt RW, Goldblum JR, Guillem JG, Kahi CJ, Kalady MF, et al: Serrated lesions of the colorectum: Review and recommendations from an expert panel. Am J Gastroenterol 107: 1315-1329, 2012.

7. Worthley DL and Leggett BA: Colorectal cancer: Molecular features and clinical opportunities. Clin Biochem Rev 31: 31-38, 2010. 
8. McCubrey JA, Steelman LS, Abrams SL, Lee JT, Chang F, Bertrand FE, Navolanic PM, Terrian DM, Franklin RA, D'Assoro AB, et al: Roles of the RAF/MEK/ERK and PI3K/PTEN/AKT pathways in malignant transformation and drug resistance. Adv Enzyme Regul 46: 249-279, 2006.

9. Van Cutsem E, Köhne CH, Láng I, Folprecht G, Nowacki MP, Cascinu S, Shchepotin I, Maurel J, Cunningham D, Tejpar S, et al Cetuximab plus irinotecan, fluorouracil, and leucovorin as first-line treatment for metastatic colorectal cancer: Updated analysis of overall survival according to tumor KRAS and BRAF mutation status. J Clin Oncol 29: 2011-2019, 2011.

10. Modest DP, Ricard I, Heinemann V, Hegewisch-Becker S, Schmiegel W, Porschen R, Stintzing S, Graeven U, Arnold D, von Weikersthal LF, et al: Outcome according to KRASNRAS- and BRAF-mutation as well as KRAS mutation variants: Pooled analysis of five randomized trials in metastatic colorectal cancer by the AIO colorectal cancer study group. Ann Oncol 27: 1746-1753, 2016.

11. Andreatos N, Ronnekleiv-Kelly S, Margonis GA, Sasaki K, Gani F, Amini N, Wilson A and Pawlik TM: From bench to bedside: Clinical implications of KRAS status in patients with colorectal liver metastasis. Surg Oncol 25: 332-338, 2016.

12. Bartel DP: MicroRNAs: Genomics, biogenesis, mechanism, and function. Cell 116: 281-297, 2004

13. Lujambio A and Lowe SW: The microcosmos of cancer Nature 482: 347-355, 2012.

14. Rusek AM, Abba M, Eljaszewicz A, Moniuszko M, Niklinski J and Allgayer H: MicroRNA modulators of epigenetic regulation, the tumor microenvironment and the immune system in lung cancer. Mol Cancer 14: 34, 2015.

15. Lee SC, Tan HT and Chung MC: Prognostic biomarkers for prediction of recurrence of hepatocellular carcinoma: Current status and future prospects. World J Gastroenterol 20: 3112-3124, 2014.

16. Liu Y, Chen X, Cheng R, Yang F, Yu M, Wang C, Cui S, Hong Y, Liang $\mathrm{H}$, Liu M, et al: The Jun/miR-22/HuR regulatory axis contributes to tumourigenesis in colorectal cancer. Mol Cancer 17: 11, 2018.

17. Chai J, Guo D, Ma W, Han D, Dong W, Guo H and Zhang Y: A feedback loop consisting of RUNX2/LncRNA-PVT1/miR-455 is involved in the progression of colorectal cancer. Am J Cancer Res 8: 538-550, 2018.

18. Huang L, Cai JL, Huang PZ, Kang L, Huang MJ, Wang L and Wang JP: miR19b-3p promotes the growth and metastasis of colorectal cancer via directly targeting ITGB8. Am J Cancer Res 7: 1996-2008, 2017

19. Zhang B, Pan X, Cobb GP and Anderson TA: microRNAs as oncogenes and tumor suppressors. Dev Biol 302: 1-12, 2007.

20. Schetter AJ, Okayama H and Harris CC: The role of microRNAs in colorectal cancer. Cancer J 18: 244-252, 2012.

21. Baraniskin A, Birkenkamp-Demtroder K, Maghnouj A, Zöllner H, Munding J, Klein-Scory S, Reinacher-Schick A, Schwarte-Waldhoff I, Schmiegel W and Hahn SA: miR-30a-5p suppresses tumor growth in colon carcinoma by targeting DTL. Carcinogenesis 33: 732-739, 2012.

22. Braun CJ, Zhang X, Savelyeva I, Wolff S, Moll UM, Schepeler T, Ørntoft TF, Andersen CL and Dobbelstein M: p53-responsive micrornas 192 and 215 are capable of inducing cell cycle arrest. Cancer Res 68: 10094-10104, 2008.

23. Wang H, Cao F, Li X, Miao H, E J, Xing J and Fu CG: miR-320b suppresses cell proliferation by targeting c-Myc in human colorectal cancer cells. BMC Cancer 15: 748, 2015.

24. O'Donnell KA, Wentzel EA, Zeller KI, Dang CV and Mendell JT: c-Myc-regulated microR NAs modulate E2F1 expression. Nature 435: 839-843, 2005

25. Johnson SM, Grosshans H, Shingara J, Byrom M, Jarvis R, Cheng A, Labourier E, Reinert KL, Brown D and Slack FJ: RAS is regulated by the let-7 microRNA family. Cell 120: 635-647, 2005 .

26. Forzati F, De Martino M, Esposito F, Sepe R, Pellecchia S, Malapelle U, Pellino G, Arra C and Fusco A: miR-155 is positively regulated by $\mathrm{CBX} 7$ in mouse embryonic fibroblasts and colon carcinomas, and targets the KRAS oncogene. BMC Cancer 17: 170, 2017

27. Lagos-Quintana M, Rauhut R, Yalcin A, Meyer J, Lendeckel W and Tuschl T: Identification of tissue-specific microRNAs from mouse. Curr Biol 12: 735-739, 2002.
28. Michael MZ, O' Connor SM, van Holst Pellekaan NG, Young GP and James RJ: Reduced accumulation of specific microRNAs in colorectal neoplasia. Mol Cancer Res 1: 882-891, 2003.

29. Akao Y, Nakagawa $Y$ and Naoe T: MicroRNA-143 and -145 in colon cancer. DNA Cell Biol 26: 311-320, 2007.

30. Ng EK, Tsang WP, Ng SS, Jin HC, Yu J, Li JJ, Röcken C, Ebert MP, Kwok TT and Sung JJ: MicroRNA-143 targets DNA methyltransferases $3 \mathrm{~A}$ in colorectal cancer. Br J Cancer 101: 699-706, 2009

31. Livak KJ and Schmittgen TD: Analysis of relative gene expression data using real-time quantitative PCR and the 2(-Delta Delta C(T)) method. Methods 25: 402-408, 2001

32. Esau C, Kang X, Peralta E, Hanson E, Marcusson EG, Ravichandran LV, Sun Y, Koo S, Perera RJ, Jain R, et al: MicroRNA-143 regulates adipocyte differentiation. J Biol Chem 279: 52361-52365, 2004

33. Lewis BP, Shih IH, Jones-Rhoades MW, Bartel DP and Burge CB: Prediction of mammalian microRNA targets. Cell 115: 787-798, 2003.

34. Griffiths-Jones S, Grocock RJ, van Dongen S, Bateman A and Enright AJ: miRBase: microRNA sequences, targets and gene nomenclature. Nucleic Acids Res 34 (Database Issue): D140-D144, 2006

35. Eslamizadeh S, Heidari M, Agah S, Faghihloo E, Ghazi H, Mirzaei A and Akbari A: The role of microRNA signature as diagnostic biomarkers in different clinical stages of colorectal cancer. Cell J 20: 220-230, 2018.

36. Wang CJ, Zhou ZG, Wang L, Yang L, Zhou B, Gu J, Chen HY and Sun XF: Clinicopathological significance of microRNA-31, -143 and -145 expression in colorectal cancer. Dis Markers 26: 27-34, 2009.

37. Ahmad I, Singh LB, Yang ZH, Kalna G, Fleming J, Fisher G, Cooper C, Cuzick J, Berney DM, Møller H, et al: Mir143 expression inversely correlates with nuclear ERK 5 immunoreactivity in clinical prostate cancer. Br J Cancer 108: 149-154, 2013

38. Shen JZ,Zhang YY,Fu HY, Wu DS and Zhou HR: Overexpression of microRNA-143 inhibits growth and induces apoptosis in human leukemia cells. Oncol Rep 31: 2035-2042, 2014.

39. Johannessen C, Moi L, Kiselev Y, Pedersen MI, Dalen SM, Braaten T and Busund LT: Expression and function of the miR-143/145 cluster in vitro and in vivo in human breast cancer. PLoS One 12: $\mathrm{e} 0186658,2017$.

40. Pichler M, WinterE, Stotz M, Eberhard K, Samonigg H, Lax S and Hoefler G: Down-regulation of KRAS-interacting miRNA-143 predicts poor prognosis but not response to EGFR-targeted agents in colorectal cancer. Br J Cancer 106: 1826-1832, 2012.

41. Friday BB and Adjei AA: K-ras as a target for cancer therapy. Biochim Biophys Acta 1756: 127-144, 2005.

42. Forrester K, Almoguera C, Han K, Grizzle WE and Perucho M: Detection of high incidence of K-ras oncogenes during human colon tumorigenesis. Nature 327: 298-303, 1987.

43. Haliassos A, Chomel JC, Grandjouan S, Kruh J, Kaplan JC and Kitzis A: Detection of minority point mutations by modified PCR technique: A new approach for a sensitive diagnosis of tumor-progression markers. Nucleic Acids Res 17: 8093-8099, 1989.

44. Brummelkamp TR, Bernards R and Agami R: Stable suppression of tumorigenicity by virus-mediated RNA interference. Cancer Cell 2: 243-247, 2002.

45. Smakman N, Veenendaal LM, van Diest P, Bos R, Offringa R, Borel Rinkes IH and Kranenburg O: Dual effect of Kras(D12) knockdown on tumorigenesis: Increased immune-mediated tumor clearance and abrogation of tumor malignancy. Oncogene 24: 8338-8342, 2005.

46. Su J, Liang H, Yao W, Wang N, Zhang S, Yan X, Feng H, Pang W, Wang Y, Wang X, et al: miR-143 and miR-145 regulate IGF1R to suppress cell proliferation in colorectal cancer. PLoS One 9: e114420, 2014

47. Hsu SD, Lin FM, Wu WY, Liang C, Huang WC, Chan WL, Tsai WT, Chen GZ, Lee CJ, Chiu CM, et al: miRTarBase: A database curates experimentally validated microRNA-target interactions. Nucleic Acids Res 39 (Database issue): D163-D169, 2011. 